\title{
EFFECTS OF CHANGING SCALES ON LANDSCAPE PATTERNS AND SPATIAL MODELING UNDER URBANIZATION
}

\author{
Jinming YANG ${ }^{1}$, Shimei LI ${ }^{*}$, Jingwei $\mathrm{XU}^{2}$, Xiaojie $\mathrm{WANG}^{3}$, Xiaoguang $\mathrm{ZHANG}^{4}$ \\ ${ }^{1}$ College of Landscape Architecture and Forestry, Qingdao Agricultural University, \\ Qingdao, 266109 Shandong, China \\ ${ }^{2}$ Shandong Academy of Forestry, Jinan, 250014 Shandong, China \\ ${ }^{3}$ Yantai Institute of Coastal Zone Research, Chinese Academy of Sciences, Yantai, 264003 Shandong, China \\ ${ }^{4}$ College of Resources and Environment, Qingdao Agricultural University, Qingdao, 266109 Shandong, China
}

Received 08 October 2019; accepted 29 January 2020

\author{
Highlights: \\ This study quantifies the block scale effect on landscape patterns and GWR models. \\ Finer block scale can exhibit more landscape pattern details of urbanization. \\ $>$ Odd-numbered block has stronger explanatory power than adjacent numbered block. \\ The driving factors have the lowest explanatory power on PD than others. \\ The local parameters of GWR models are scale-dependent.
}

\begin{abstract}
Spatial scale is an eternal topic in landscape pattern related analysis. This paper examined the spatial scale effect of landscape pattern changes and their relationships with urbanization indicators in Qingdao using a series of sampling blocks. The results indicated that, with the increasing block scale, the mean patch density and aggregation within a block decreased, whereas the diversity increased. Furthermore, the expanding scale amplified the mean change ratio of landscape metrics and eliminated local drastic changes and regional variation trends along an urban-to-rural gradient, which would be obvious at a finer block scale. Meanwhile, the adjusted $\mathrm{R}^{2}$ of GWR (Geographically Weighted Regression) models increased with an increasing block size, especially when the block scale changed from $1 \mathrm{~km}$ to $5 \mathrm{~km}$. Odd-numbered block scales performed better than even-numbered block scales.
\end{abstract}

Keywords: spatial scale, block size, urbanization, landscape patterns, geographically weighted regression (GWR).

\section{Introduction}

People living in urban areas worldwide has reached 50\% and this will increase to $65 \%$ by 2050 (Ali et al., 2019). The aggregation of population has caused tremendous urban land extension (Yang et al., 2019) and accompanied social and economic activities (Marull et al., 2015). At the same time, this rural-urban process can lead to many environmental issues (Sun et al., 2018). For example, transformation from forest or cultivated land to impervious land, which occurs under urbanization, will affect climate regulation and cause an urban heat island effect (Zhou \& Cao, 2020). Expansion of built-up land and a more concentrated population can also cause resource crises, atmospheric contamination, traffic jams, noise pollution, food supply reductions and carbon sequestration declines
(Li et al., 2016; Li et al., 2017). These problems have been largely studied from the perspective of landscape patterns (Li et al., 2017; Zhang \& Su, 2016) and are often described using landscape metrics (Dadashpoor et al., 2019; Jiao et al., 2019; Shen et al., 2019; Yang et al., 2019).

However, the landscape pattern is scale-dependent (Wu et al., 2002). For the studies of landscape ecology, the choice of scale is an indispensable and important link (Feng et al., 2018; Li et al., 2017; Zhou \& Cao, 2020). Large scale often ignores some subtle features, while small scale would be lack of global perspective. Numerous studies have investigated various scale effects on landscape patterns using contrived and real data (Tian et al., 2019; Wu, 2004; Wu et al., 2002). These spatial scales can be classified into two types: a change in grain size, and a change

*Corresponding author. E-mail: li_shimei@163.com 
in extent. Grain is the spatial resolution of the data (i.e., the area represented by each cell). When analyzing this effect on landscape patterns, the original spatial resolution is resampled at multiple scales. For example, $\mathrm{Wu}$ et al. systematically changed four landscapes' grain sizes from $1 \times 1$ to $100 \times 100$ pixels following the majority rule and investigated the influence of changing the spatial resolution on 19 commonly used landscape metrics $(\mathrm{Wu}$ et al., 2002). Meanwhile, regular grids or blocks generated from GIS fishnets have been adopted to study spatial heterogeneity of landscape patterns (Du et al., 2014; Li et al., 2017; Su et al., 2011). Feng et al. compared landscape metrics measured in moving windows with different sizes (Feng et al., 2018), which takes cells into consideration repeatedly during the metric calculation process and finally generates maps of metrics with a spatial resolution that is the same as that of the original map. In addition to these regularly changing extents, irregular boundaries, such as political, metropolitan, ecological and watershed boundaries, have been used to evaluate the effect of the extent on landscape patterns (Su et al., 2014; Torres et al., 2016).

The scale effect of landscape pattern will be introduced to spatial relationships between landscape metrics and various ecological indicators which was often explored by geographically weighted regression (GWR) (Alibakhshi et al., 2019; Li \& Zhao, 2019; Li et al., 2017; Luo \& Peng,
2016; Yang et al., 2019). Studies have demonstrated that three aspects of scale effects existed in GWR models (Yang et al., 2019): (1) the kernel bandwidth, which determines the weight; (2) the block scale selected when calculating the landscape metrics; and (3) selection of the window size when analyzing local variance. Although these scale effects have been taken into consideration in most of the works related to GWR, a systematic understanding of the block scale effect in GWR modeling is still lacking. Therefore, further research should be conducted to clarify the block scale effect on GWR results.

In order to check the effect of block scale on GWR models, we built relationships between landscape metrics and urbanization indicators. Qingdao city was taken as a case study because of its rapid economic development and the succedent land use changes. When building models, the change in landscape metrics is taken as an induced variable, and the independent variables include socioeconomic data (gross domestic product and population density) and the human activity intensity (nighttime light data). Specifically, the primary goals of this study are to (1) recognize the land-use and landscape pattern changes between 1995 and 2010 in Qingdao, (2) investigate the effects of the block scale on landscape patterns systematically and (3) check the influences of a series of block scale on GWR models between landscape pattern changes and urbanization.

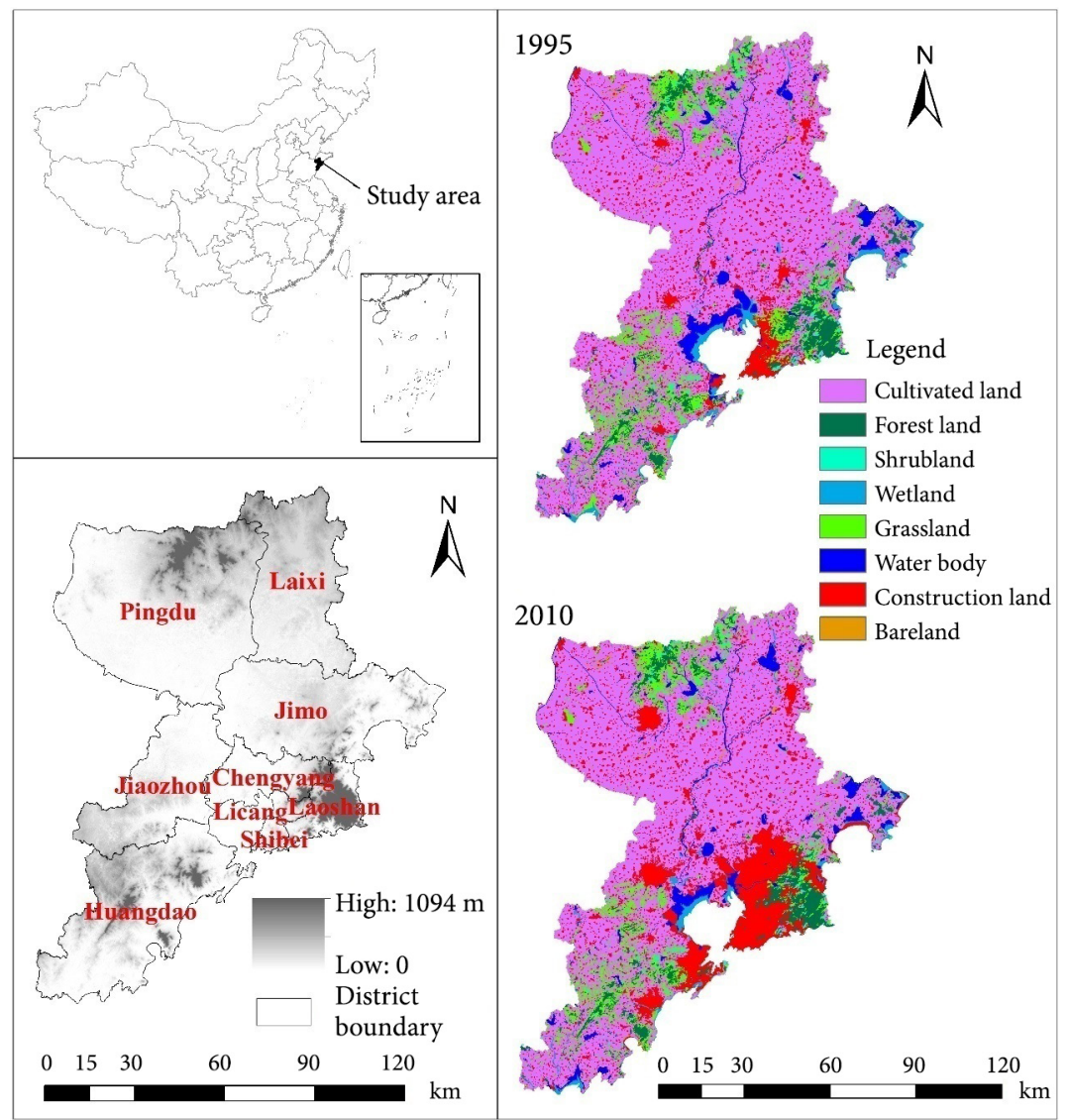

Figure 1. Location of Qingdao and its land-use map from 1995 to 2010 


\section{Study area and data sources}

\subsection{Study area}

Qingdao is a city with quick economic development in Shandong province, which lies on the east coast of China with latitude stretches from $35^{\circ} 35^{\prime}$ to $37^{\circ} 09^{\prime} \mathrm{N}$ and longitude from $119^{\circ} 30^{\prime}$ to $121^{\circ} 00^{\prime} \mathrm{E}$ (Figure 1). The city encompasses a total land area of $11,282 \mathrm{~km}^{2}$ and had a population of 9 million in 2017. Situated on the Yellow Sea in the east and south, Qingdao possesses a representative marine climate with an annual average temperature of $12.4{ }^{\circ} \mathrm{C}$ and an average precipitation of $662 \mathrm{~mm}$ (Peng et al., 2018). From 1995 to 2010, the total residential population of Qingdao increased from 6.78 million to 8.71 million. Gross domestic product and its per capita in Qingdao have experienced rapid growth since 1990, with the growth rates exceeding one digit from 1998 to 2009 apart from the global financial crisis years (2007-2008) (Zhang \& Rasiah, 2013). Qingdao has 6 districts (Shinan, Shibei, Licang, Laoshan, Huangdao and Chengyang) and comprises five county-level cities (Jiaonan, Jiaozhou, Jimo, Pingdu and Laixi) (Figure 1).

\subsection{Data sources}

Three datasets were used in our study: land-use/cover (LUCC) dataset, spatial socioeconomic dataset and nighttime light (NTL) dataset. The LUCC data were used to calculate landscape metrics and analyze landscape patterns in Qingdao. The socioeconomic and NTL data were taken as indicators of urbanization. The acquisition methods and related details for these data are listed below.

The LUCC and the socioeconomic data for Qingdao in1995 and 2010 were collected from the Resource and Environment Data cloud platform, Chinese Academy of Sciences (Xu et al., 2018). Their spatial resolutions are $30 \mathrm{~m}$ and $1 \mathrm{~km}$, respectively. The original LUCC types were reclassified into eight categories (Figure 1). Among these land types, the water body type includes the original secondary type of rivers, lakes, reservoirs and ponds, whereas the wetland type is composed of mud flat, bottomland and marshland.

The values of each socioeconomic raster are the gross domestic product (GDP) and population per $\mathrm{km}^{2}$ (POP), which were calculated through interpolation considering the county's differentiation of GDP and demographic data.

The version 4 NTL datasets in 1995 and 2010 were derived from the US Government's Defense Meteorological Satellite Program Operational Line Scan System (DMSPOLS) Nighttime Lights remote sensing imagery (Defense Meteorological Satellite Program, n.d.). These data were provided in 30-arc-second grids with pixel values varying from 0 to 63 . The NTL data have been effectively applied to estimate the dynamics of urban sprawl because the average pixel values are mainly from lights in cities, towns and places with lasting illumination (Baugh et al., 2010; Zhang \& Seto, 2011). Thus, we designated this dataset an indicator of the human activity intensity. However, some calibrations must be adopted before using the time series of these data due to the lack of on-board calibration and inter calibration, which will result in non-continuity and non-comparability (Elvidge et al., 2009; Liu et al., 2012). To improve the continuity and comparability between different years and different satellite data, we adopted the methods developed by Liu et al. (2012) to preprocess the NTL datasets in this study. First, a second-order regression model for inter calibration was used to improve the comparability of NTL data between different years; second, information from two satellites in the same year was utilized to eliminate as table pixels in that year; and finally, inconsistencies in pixels in the multi-year dataset were corrected through an inter-annual series correction method. After the above steps, the preprocessed global NTL datasets from 1995 and 2010 were projected onto a Universal Transverse Mercator projection (UTM zone $51 \mathrm{~N}$ ) and resampled to a $1 \mathrm{~km}$ spatial resolution. Then, the administrative boundaries of Qingdao were used to extract the NTL datasets.

\section{Methods}

\subsection{Calculation of landscape metrics}

When analyzing landscape patterns, the selection of appropriate metrics is very important. To allow comparisons with previous studies and avoid autocorrelation between variables (Li et al., 2017; Riitters et al., 1995), four landscape-level metrics were used in this study: 1) the patch density (PD), which is the patch number per square kilometer and is an indicator of fragmentation and aggregation of a landscape; 2) the edge density (ED), which is calculated as the summation of all edge lengths per hectare and indicates a more irregular shape of patches with a larger value; 3) Shannon's diversity index (SHDI), which can reflect the heterogeneity of the landscape with a value equal to zero when only one patch exists; the value will increase when the patch type number increases and/or when different patch types becomes more equitably distributed; and 4) the aggregation index (AI), which can provide knowledge of landscape structure and is helpful for guiding the urbanization process toward sustainability.

\subsection{Generating of the series of blocks}

To systematically investigate the effects of changing scale on the landscape patterns and GWR models, several steps were conducted. First, a series of fishnets with pixel sizes of $0.9 \mathrm{~km}$ (hereafter $1 \mathrm{~km}$, the round method is the same with other decimal scales), $2.1 \mathrm{~km}, 3 \mathrm{~km}, 3.9 \mathrm{~km}, 5.1 \mathrm{~km}$, $6 \mathrm{~km}, 6.9 \mathrm{~km}, 8.1 \mathrm{~km}$ and $9 \mathrm{~km}$ were created by the Fishnet tool in ArcGIS 10.3. The reason for using the decimal scale was that these sizes were integral multiple of 30 which is the spatial resolution of the LUCC. The scale was restricted to $9 \mathrm{~km}$ because a scale larger than $9 \mathrm{~km}$ would contain too few blocks to conduct the analysis. Blocks with less than half of their land located in the boundary of Qingdao were deleted. 
Second, nine fishnets were intersected with the LUCC maps in 1995 and 2010 to split the raster into tiles associated with each block. This step was conducted using the Split Raster tool in ArcGIS 10.3.

Third, four landscape-level metrics for each tile of the nine fishnets were computed in the Fragstats 4.2 software using the batch method. Then, the results were exported and joined to the attribute table of each fishnet dataset by tile names.

Finally, the differentiation between the 1995 and 2010 changes was computed as a new attribute field for each block scale through Eq. (1):

$$
R_{i}=\frac{M_{2010 i}-M_{1995 i}}{M_{1995 i}} \times 100,
$$

where: $R_{i}$ is the change ratio of the landscape metrics in block $i$, and $M_{1995 i}$ and $M_{2010 i}$ respectively represent the value of the metrics of location $i$ in 1995 and 2010.

\subsection{Processing of urbanization indicator}

The three urbanization indicators (GDP, POP and NTL) were handled based on the following process: 1) First, the original datasets were resampled to a resolution of $100 \mathrm{~m}$, which could be divided evenly into nine block sizes; 2) Second, 9 fishnets were intersected with 3 datasets to extract the exact boundaries of the fishnets; 3 ) Third, the average values of the pixels within each block were calculated and normalized based on the min-max standardized method; 4) Finally, the normalized urbanization indicators in 1995 and 2010 were extracted and joined to the attribute tables of the 9 fishnet datasets.

\subsection{Building of GWR model}

In this study, the GWR model was adopted in the regression analysis to identify the spatial variation of the relationships between landscape patterns and urbanization indicators. GWR is a local linear regression method that calculates the regression coefficients at each location and outputs a series of location-specific coefficients, including residuals, estimates and local coefficients (Fotheringham et al., 2002; Yang et al., 2019). Under the assumption that closer observations exert more significant influences, the GWR model restricts the spatial scope of dependence by using the kernel bandwidth and then calculates weights for all observations via a distance decay function. The formulation of the GWR model can be presented as Eq. (2):

$$
y_{j}=\beta_{0}\left(u_{j}, v_{j}\right)+\sum_{i}^{k} \beta_{i}\left(u_{j}, v_{j}\right) x_{i j}+\varepsilon_{j},
$$

where: $u_{j}$ and $v_{j}$ are respectively the abscissa and ordinate of location $j, \beta_{0}\left(u_{j}, v_{j}\right)$ and $\beta_{i}\left(u_{j}, v_{j}\right)$ respectively, represent the intercept and the regression coefficient $x_{i j}$, and $\varepsilon_{j}$ represents the deviation.

The Gaussian distance decay function was used to calculate the spatial weight matrix through Eq. (3):

$$
w_{i j}=\exp \left(-\frac{d_{i j}^{2}}{b^{2}}\right) .
$$

In the above equation, $w_{i j}$ denotes the weight of observation $i$ with respect to neighborhood location $j, d_{i j}$ represents the Euclidean distance between two locations, and $b$ represents the kernel bandwidth. The kernel bandwidth is the decay distance for the weight of observation points, which represents on intrinsic scale (Gao \& $\mathrm{Li}$, 2011). A greater kernel band width will make the relationships more global and cause the spatially estimated coefficients to be more stationary (Li et al., 2010).

When observations $i$ and $j$ are located in the same place, then the weight coefficient equals 1; conversely, the weight value decays close to zero when the distance between them increases toward the spatial scope of the kernel bandwidth. A coarser kernel bandwidth leads to slower weight decreases with distance and generates more global relationships (Li et al., 2010). The kernel function of the GWR model includes two types: fixed and adaptive. In this study, we adopted the fixed type of kernel bandwidth as the density of the sampling points because the grid data used in this study were uniform.

All regression models were performed using the GWR tool in ArcGIS 10.3 (Environmental Systems Research Institute, Redlands, CA, USA). The change in each landscape metric was used as dependent variable, and one of the urbanization indicators (GDP, POP and NTL) was used as independent variable. We selected only one indicator as an explanatory variable because the urbanization indicators might present multicollinearity, which would impact the analysis of scale effects (Dormann et al., 2013, 2007).

\section{Results}

\subsection{Land-use changes from 1995 to 2010}

Qingdao experienced significant urban expansion, which caused fundamental land-use changes from 1995 to 2010. Revealed from the LUCC conversion matrix (Table 1), the area for the changed land was 95, 675.7 ha. This indicated that over $8.59 \%$ of the land changed, with a yearly changed ratio of $0.57 \%$. The mostly changed land-use types were cultivated land, grassland, construction land and waterbody, which accounted for $79.62 \%$ of the total changes. Specifically, the construction land expanded by 84,011 ha which was $66.51 \%$ of the changes during this period. However, other land-use types declined differently, with the area of the cultivated land declined by $59,266.5$ ha (7.98\%), the grassland decreased by $15,524.6$ ha (1.39\%) and the wetland area decreased by $6,889.1$ ha $(0.62 \%)$. The results also showed that the cultivated land and grassland had greater contributions to urban land than other types, contributing up to approximately $64.73 \%$ and $15.47 \%$, respectively. This result was consistent with our previous study (Yang et al., 2019). Among the increments of 84, 011 ha of built-up land, 54, 623 ha came from cultivated land and 13, 059 ha were converted from grassland. Some 
Table 1. Land-use/cover conversion matrix from 1995 to 2010 in Qingdao (in ha)

\begin{tabular}{|c|c|c|c|c|c|c|c|c|c|}
\hline \multirow{2}{*}{1995} & \multicolumn{8}{|c|}{2010} & \multirow{2}{*}{$\begin{array}{l}1995 \\
\text { Total }\end{array}$} \\
\hline & Cultivated & Forest & Shrubland & Wetland & Grassland & Waterbody & Construction & Bareland & \\
\hline Cultivated & $682,656.4$ & 372.2 & 1 & $1,173.2$ & 15.4 & $3,705.9$ & 54,623 & 0.9 & 742,548 \\
\hline Forest & 4.3 & $55,108.1$ & & 19.8 & 0.9 & 78.8 & $1,336.9$ & 0.1 & $56,548.9$ \\
\hline Shrubland & 0.8 & & 11,517 & 0.1 & 0.1 & 44.9 & 825.9 & & $12,388.8$ \\
\hline Wetland & 12.3 & 0.6 & 0.6 & $14,609.6$ & 3.2 & $2,445.2$ & $5,839.6$ & 0.2 & $22,911.3$ \\
\hline Grassland & 392.7 & 32 & 0.3 & 26.1 & $87,593.7$ & $2,038.1$ & 13,059 & & $103,141.9$ \\
\hline Water body & 138.6 & 0.3 & 0.2 & 174.7 & 1.4 & $37,551.4$ & $8,495.6$ & & $46,362.2$ \\
\hline Construction & 75 & 5.4 & & 5 & 2.6 & 289.6 & $125,927.8$ & 0.3 & $126,305.7$ \\
\hline Bareland & 1.4 & 0.2 & & 13.7 & & 208.7 & 208.9 & $3,322.6$ & $3,755.5$ \\
\hline 2010 Total & $683,281.5$ & $55,518.8$ & $11,519.1$ & $16,022.2$ & $87,617.3$ & $46,362.6$ & $210,316.7$ & $3,324.1$ & $\begin{array}{c}1,113 \\
962.3\end{array}$ \\
\hline
\end{tabular}

research in the eastern China also resulted that the farmland play a great role in transformation to construction land (Deng et al., 2009; Shen et al., 2019; Su et al., 2011), which indicated that the sprawl of urban areas embezzled large amount of agricultural land.

\subsection{Synopticanalysis of landscape patterns}

Refer to Figure 2, the landscape level metrics of Qingdao changed slightly from 1995 to 2010 . The combination effect of a decrease in the PD and increase in SHDI indicated that the average patch numbers increased and the distribution became more equitable among the different land types. These landscape changes were the result of conversion from the predominantly cultivated land to an urban landscape, which made the numbers and distributions of the different patch types more even. The PD and ED decreased by $2.6 \%$ and 3.2 , respectively, indicated a fragmentation which was the most common result due to urbanization (Dadashpoor et al., 2019; Yang et al., 2019).

\subsection{Spatial-temporal changes in the landscape pattern at different block scales}

The spatial patterns of the change ratios for the four metrics between 1995 and 2010 in Qingdao were demonstrated at each block scale in Figure 3. In general, the value of landscape metrics for most of the blocks remain unchanged (the yellow regions shown in Figure 3) at the $1 \mathrm{~km}$ scale. The PD significantly decreased in the Shinan, Shibei, Licang and Chengyang districts. Most patches in these places were already built up in 1995. Under the urbanization process, the isolated patches were connected. Consequently, the number of patches decreased, and thus the PD decreased. The increase in the PD, especially those that increased by more than $40 \%$, was mainly distributed in Jiaozhou city, eastern Chengyang district and dotted around villages. The main reason for these was that a large amount of cultivated land was transformed to construction land, creating a more fragmented landscape in the

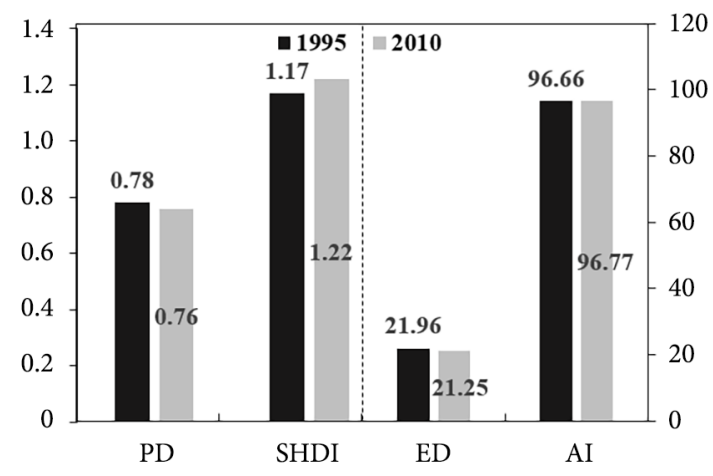

Figure 2. Changes in landscape level metrics of PD (patch density), SHDI (Shannon's diversity index), ED (edge density) and AI (aggregation index) at the whole region scale

block. The fragmentation in the urban-rural fringe areas caused by urbanization were also found in other areas, like Xi'an (Hou et al., 2020), Beijing (Li et al., 2019), North America (Koch et al., 2019), and Leiria City (Barros et al., 2018).

The evolutionary characteristics of the ED and SHDI in space from 1995 to 2010 coincided with PD. Driven by urbanization, the built-up areas became further developed and formed larger patches. As a result, the ED and SHDI significantly decreased in these areas. In northern Jiaozhou city, eastern Chengyang district and the circumjacent built-up areas, the ED and SHDI increased significantly. These regions witnessed severe urbanization, and other land types were transformed to construction land. The changes in AI behavior were in the opposite directions as those of the other metrics. In Shinan, Shibei, Licang and the rural towns of Pingdu, Jimo, Jiaozhou and Jiaonan district, the AI increased significantly. This increase was caused by replacing the initial cultivated land and forest land with built-up land. A significant decrease was observed in the surrounding areas, and no obvious changes occurred in the remaining areas. 


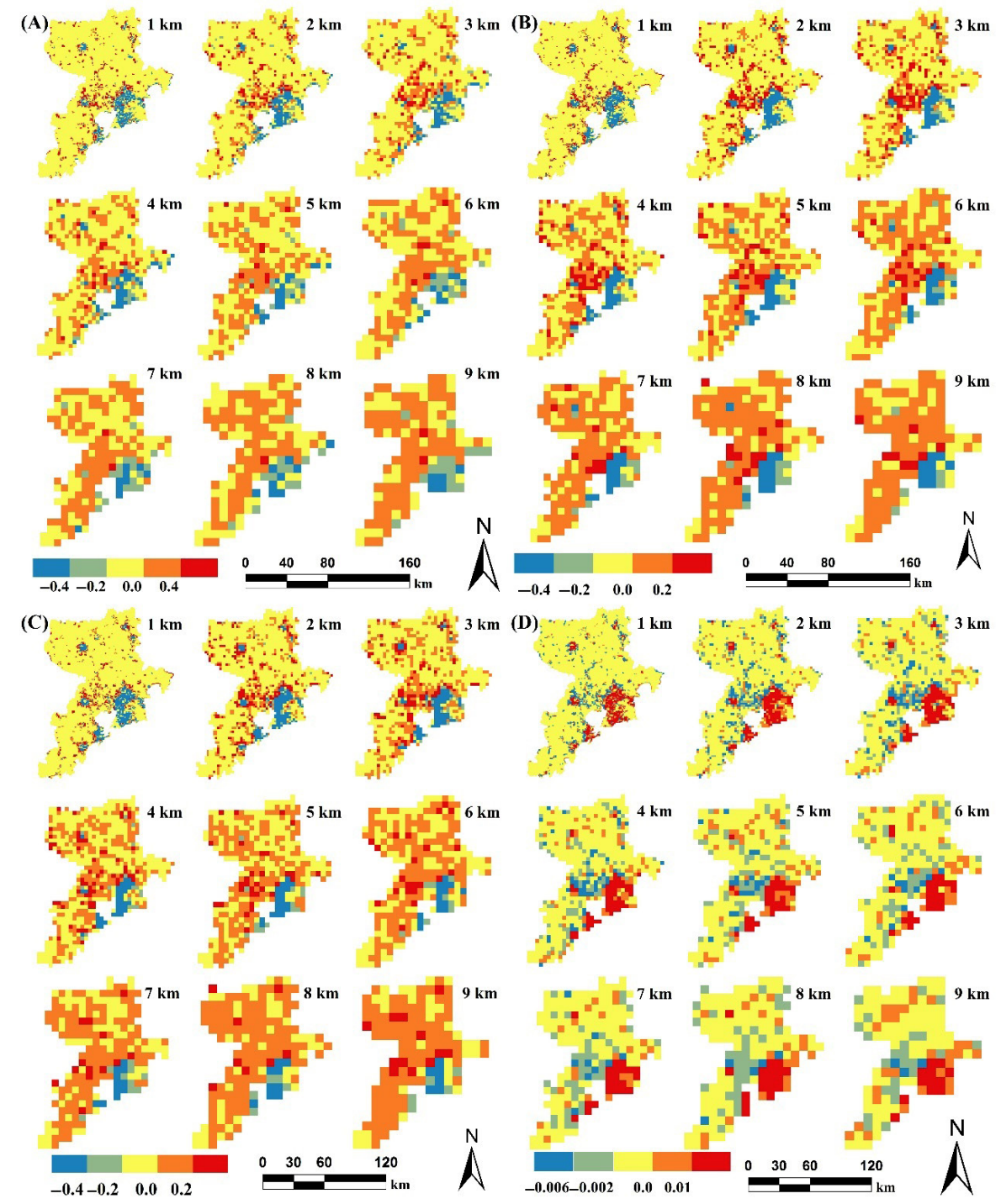

Figure 3. Spatial patterns of change ratios for landscape metrics in Qingdao at different block scales from 1995 to 2010. A, B, C and Drepresent maps of changes in the PD, ED, SHDI and AI, respectively.

Here, $1 \mathrm{~km}$ represents $0.9 \mathrm{~km}$ of the block size (i.e., the pixel size). Further details are provided in Section 2.1

The influence of the block scale on the spatial changes of all landscape metrics was obvious. With the expansion of block scale, the proportion of changed blocks increased and the number of blocks with great changes decreased. As shown in Figure 3A, the percentage of blocks with drastic changes (decreased or increased by more than $40 \%$ ) decreased with the increase of scale. Specifically, compared with $1 \mathrm{~km}$ block scale, blocks with PD changed from $0 \%$ to $40 \%$ occupied most of the region, whereas only 7 of 140 blocks changed greater than $40 \%$. Larger blocks contained more spatial areas; therefore, the landscape metrics for the entire block area changed when tiny land cover changes occurred. In addition, great landscape metric changes in local areas were equilibrated by places with lower changes that predominated in one block area. A similar trend was found for other landscape metrics (Figure 3 B-3D).

The mean value of landscape metrics for different block scale was calculated and shown in Figure 4. With the increase of block scale, the averaged PD constantly declined approximately $76 \%$ from 3.9 to 0.9 . The mean value of AI decreased by $0.94 \%$ and $0.89 \%$ for 1995 and 2010, respectively, and the difference became larger. However, the averaged SHDI continuously increased from approximately 0.44 to 0.86 , and the mean value of ED exhibited a saddle shape in response to the increase of block size. Furthermore, the slope of curves for PD, SHDI and AI showed a constant decline.

\subsection{Effects of the spatial scale on the adjusted $R^{2}$ and local parameters ofthe GWR model}

In this study, a total of 216 GWR models between landscape metrics and urbanization indicators at different block scales were obtained (Figure 5). There into, the adjusted $\mathrm{R}^{2}$ for 161 models (74.5\%) exceeded 0.4, indicating that GWR had great power in explaining the influences of the urbanization indicators on landscape patterns. 

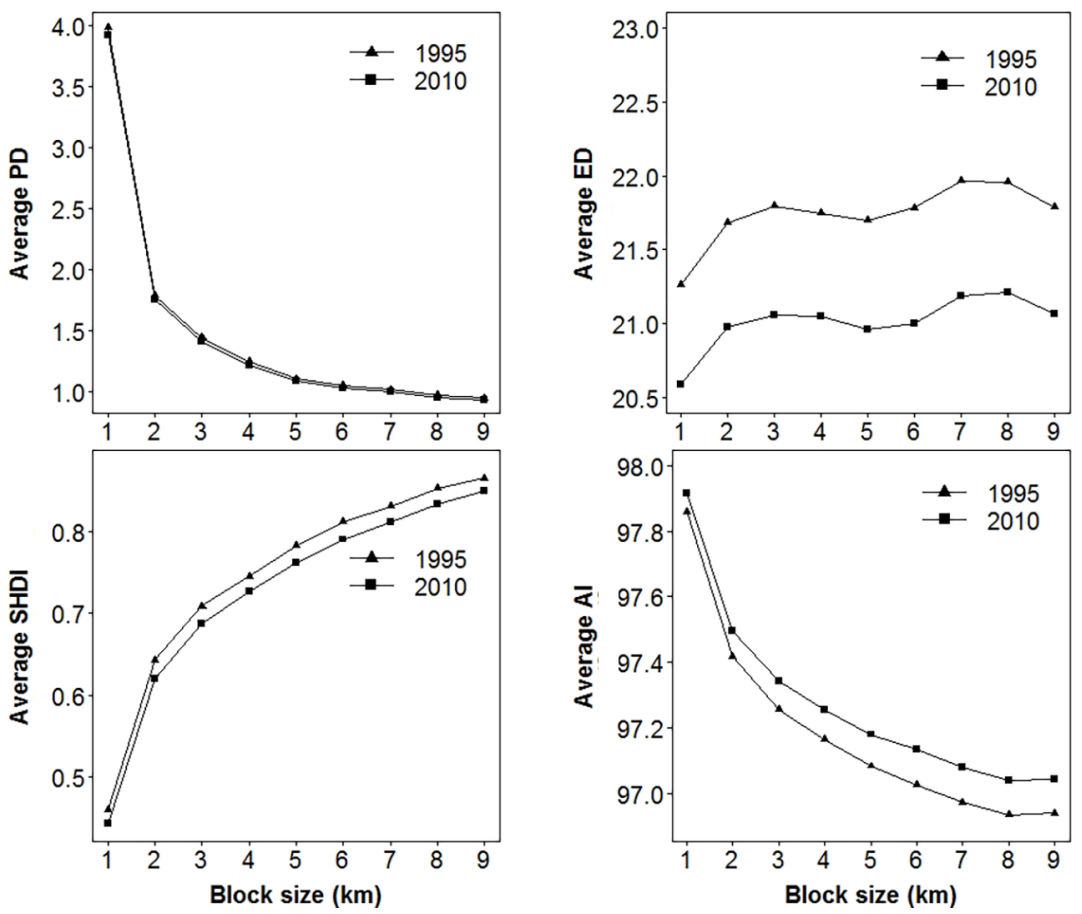

Figure 4. Averaged landscape metric changes in response to the block scale in Qingdao in 1995 and 2010
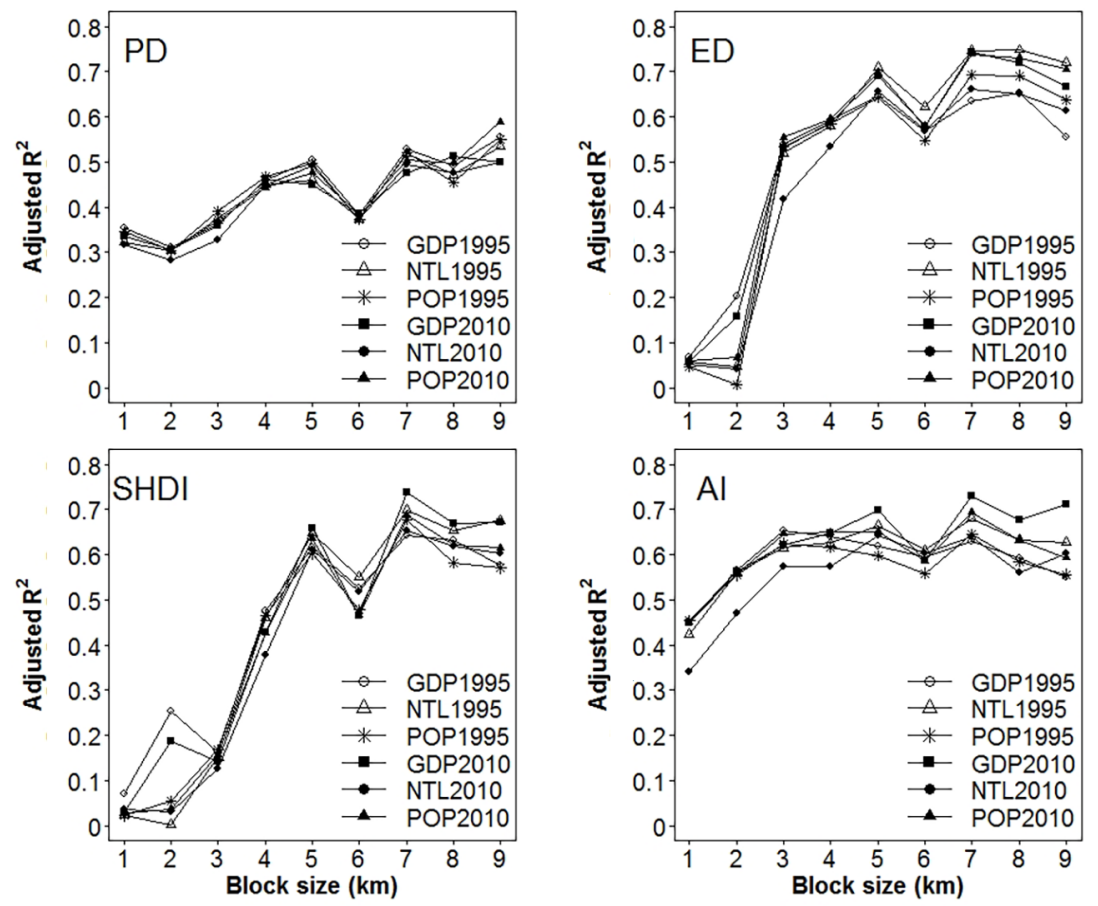

Figure 5. The effects of block scale on the performance (adjusted $\mathrm{R}^{2}$ ) of the GWR models between landscape metrics and urbanization indicators (GDP, NTL and POP).

GDP, NTL and POP represent the gross domestic product, nighttime lights and population, respectively 
Block scale had a strong influence on the GWR models, especially those explored with metrics of ED and SHDI. When the block size enlarged from 1 to $5 \mathrm{~km}$, the adjusted $\mathrm{R}^{2}$ significantly increased for the models obtained from ED and SHDI and slightly increased for the models explored with PD and AI. But, when the block scale continuously expanded to $9 \mathrm{~km}$, the adjusted $\mathrm{R}^{2}$ for most of the GWR models showed a slight wave-like trend with a sharp decrease at the $6 \mathrm{~km}$ block. Furthermore, the odd-numbered block scales seemed to have better performance than the even-numbered block scales. The reason may be that the odd-numbered scales were not integral multiples of the socioeconomic dataset which was $1 \mathrm{~km}$ spatial resolutions. So, it will borrow in $0.1 \mathrm{~km}$ to fill up a $2.1 \mathrm{~km}$ or some other block, and this added space could give more details referred to land-use changes and hence landscape changes. Among the landscape metrics, PD performed worse than the other metrics.

Due to space limitations, we only took models explored by the AI and GDP in 1995 as an example to illustrate the spatial patterns of the GWR parameters and the scale effects on local variance. As shown in Figure 6, both the coefficient and intercept have spatial heterogeneity and aggregative effects at each block scale. The larger values of coefficients mainly distributed in the settlements of Pingdu, Laixi and Jimo district. The block scale had an obvious impact on the GWR results (Figure 6). The GDP had significant positive effects on landscape pattern changes at the urban fringes of Pingdu, Laixi, Jimo, Jiaozhoucity and Laoshan district and negative effects on cultivated land in most of the regions at the $1 \mathrm{~km}$ block scale. However, with the block size increased, the spatial difference of the coefficients and intercepts decreased, some negative values transformed to positive or on the contrary especially in those places of Jiaozhou Bay and Pingdu district.

\section{Discussion}

\subsection{How does rapid urbanization impact landscape patterns?}

Aggregation of population and wealth leads to a large demand for land for housing, commerce, public infrastructure and industry. Then, the consequent construction projects progressively transform open land to built-up land. This process is known as "urban sprawl" or urbanization (Mann, 2009). Conversion between land-use types results in recombination of the environment and thus changes the landscape pattern (Dadashpoor et al., 2019). When urbanization intensified in Qingdao from 1995 to 2010, much of the cultivated land and grassland were transformed to urban land, causing the landscape pattern to become fragmented and isolated. This phenomenon was prominent in the eastern cities of China (Xiao et al., 2018). For example, in the Jing-Jin-Tang metropolitan areas, a great amount of agricultural land was transformed to built-up land, contributing approximately $74 \%$ of the newly added urban land (Tan et al., 2005). During this process, the original large patches become fragmented, and the connected corridors were destroyed (Yang et al., 2019). These landscapes will cause the biological diversity of ecosystems to descend and become fragile and unstable, which is considered unsuitable for sustainable development (Wang et al., 2019). Therefore, to balance the fused relationship between construction land and other landscapes and achieve sustainable development, landscape fragmentation issues should be considered under the process of urbanization.

\subsection{How does the landscape pattern change with the block scale?}

The scale effects of extent on landscape metrics have been well studied using artificial and real land-use datasets

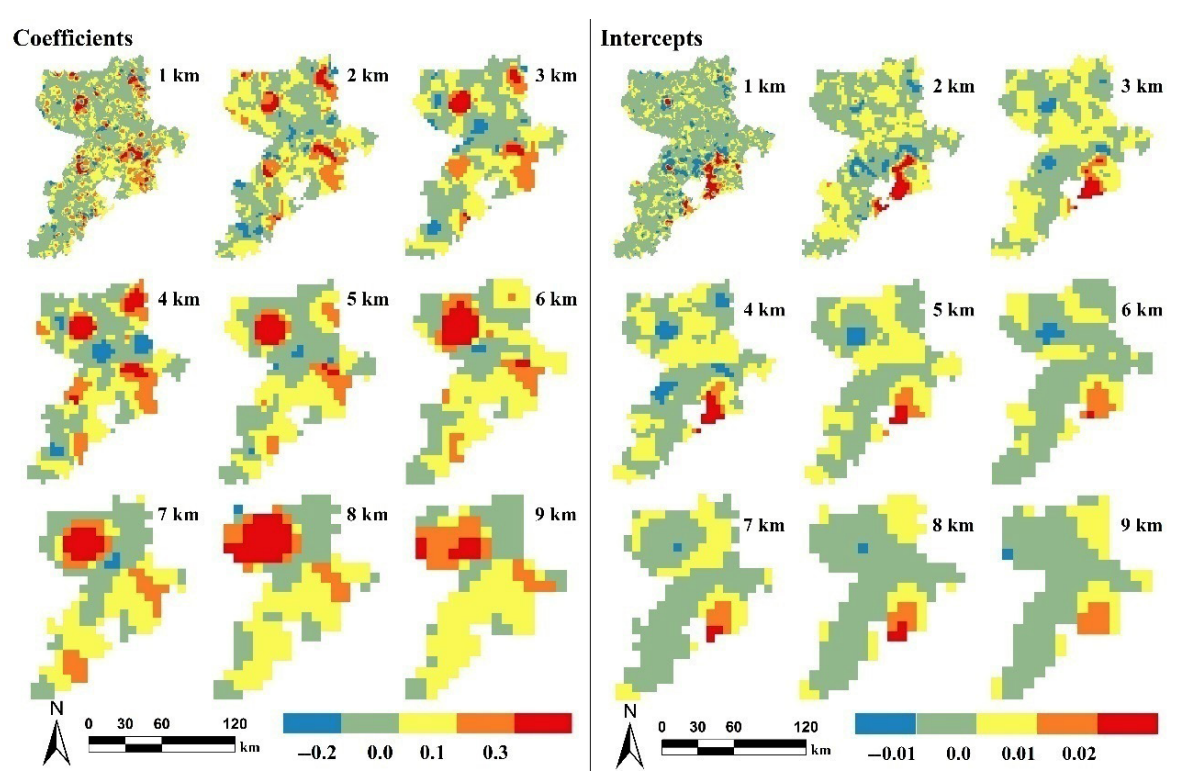

Figure 6. Scale effects on the spatial distribution of coefficients and intercepts of GWR models between the AI change ratios and GDP in 1995 
(Shen et al., 2004; Wu, 2004; Wu et al., 2002). According to these studies, three general types of responses exist with changes in extent: Type I, metrics have predictable responses with simple scaling relationships (coincide with power exponent or linear functions); Type II, metrics respond as a staircase; and Type III, metrics show erratic responses. Zhou et al. also claimed that changing spatial extent have significant impacts on the relationship between spatial pattern of urban forested areas and land surface temperature (Zhou \& Cao, 2020). What types of responses for landscape metrics with respect to block scale belong to? According to our study, SHDI, PD and AI belongs to Type I, which is in accordance with previous findings (Feng \& Liu, 2015; Wu et al., 2002). But it isn't consistent with the study of Xu et al., who demonstrated that SHDI is unpredictable across scales (Xu et al., 2019). ED is included in Type III, which has unpredictable behavior. PD, SHDI and AI are more sensitive to block scale than ED.

What block scale can better explain changes of landscape pattern? Studies have proved that different landscapes have different characteristics of pattern and respond unlikely to scale changes (Su et al., 2011; Wu et al., 2002; Xu et al., 2019; Zhou \& Cao, 2020). Therefore, specific landscape pattern, exhibited as landscape metrics, has its own appropriate scale. However, the proper block scale for GWR models under urbanization still lack of reports. As shown in our study, ED and SHDI are more sensitive to block scale than PD and AI (Figure 5). Block scale of $7 \mathrm{~km}$ is more suitable for GWR analysis associated with the landscape metrics of ED, SHDI and AI, whereas $9 \mathrm{~km}$ or greater is the most fitting scale for GWR analysis of PD. Our result is not consistent with Zhou et al. who suggested that $90 \mathrm{~m}$ was the optimal spatial scale for studying the cooling effect of forest vegetation in Shanghai's urban area (Zhou \& Cao, 2020). However, they used moving window method which is some different with our study. Another interesting result in our study is that the odd-numbered block scales performed better than even-numbered block scales (Figure 5). These characteristics can be used as GWR performance predicts when exploring the relationship between landscape metrics and urbanization indicators.

Also, previous studies have analyzed spatial changes in landscape patterns along the gradient from cities to rural areas using landscape metrics (Herold et al., 2003, 2002; Luck \& Wu, 2002; Weng, 2007). Some general trends revealed by these case studies were confirmed in our study (Figure 4). The landscape at the urban center became less diverse and fragmented due to aggregation of built-up and residential lands (i.e., there were lower PD and SHDI values, and the AI value was higher (Herold et al., 2002). Compared to those of urban centers and rural areas, the landscape diversity and fragmentation were highest at the urban fringes (Weng, 2007). Similarly, the urbanization process will also fragment urban green space (Li et al., 2019). The general trends of spatial and temporal changes in landscape patterns along the urban-to-rural gradient were obvious at finer block scales, such as the $1 \mathrm{~km}, 2 \mathrm{~km}$ and $3 \mathrm{~km}$ block scales. However, generally the trends disappeared when the block scale exceeded the area of the urban center and urban fringes. Therefore, the spatial scale is a key issue that should not be ignored when analyzing changes in landscape patterns impacted by urbanization.

\subsection{How does GWR model performedwith respect to block scale, and what are the implications?}

The scale-dependent characteristics of landscape pattern will transform to their relationships with spatial factors like urbanization indicators. We explored relationships between landscape pattern changes and urbanization using GWR method at a series of block scale to answer the question of how the block scale will affect GWR performance. The results showed that both the explaining power and the estimated parameters of these models were changing with respect to the block size (Figure 5 and 6). With the block size changing from $1 \mathrm{~km}$ to $9 \mathrm{~km}$, the adjusted $\mathrm{R}^{2}$ of models for the four metrics generally increased. Similar results were reported in the study by Gao and Li (Gao \& $\mathrm{Li}, 2011)$, in which spatial non-stationary and scaledependent relationships were found between urban landscape patterns and urban expansion. Su et al. employed local indicators to evaluate the spatial dependence of relationships between agricultural landscape changes and urbanization at two grid scales and found scale-dependent relationships too (Su et al., 2011). These findings can offer scientific references for urban planning to coordinate the relationship between urban development and landscape.

The results from this study could provide a clear comprehending of how the block scale will affect GWR model building. When studying the relationships between landscape pattern and urbanization, a proper block size could be easily determined based on our study. However, many factors play a role in the exploring of GWR, such as the quality of the geographic data source, temporal scale, selection of landscape metrics, urbanization indicators and the changing method of spatial extent. Therefore, some research work should be further done. (1) The block scale effect on GWR performance should be verified in other regions or bigger cities and to check the effect of block size that was greater than $9 \mathrm{~km}$. (2) Besides the block method of changing spatial extent, the moving windows method which also have the scale effect of extent should be studied too. (3) The scale effect on other landscape metrics and their relationships with urbanization indicators or other ecological factors should also be addressed.

The scale effect could help policy makers to check out in what spatial scales human activities could evidently change landscape pattern and do policy making accordingly. For example, the GDP, POP, and NTL could explain the landscape changes in Qingdao when the block size is greater than $4 \mathrm{~km}$ in this study. Therefore, urban development managers should have a perspective of large scale while do urban planning. Make sure that in each great 
block size avoid fragmentation of landscape especially in the urban fringe area. Large patches of forest land or grass land should be preserved during the urban sprawl. Some green belts or large parks containing large forest land, grass land and water body could also be planned. These efforts should be conducted at every specific block size to avoid excessive aggregation of built-up lands. After all, the aggregation of impervious surface and population could bring many environmental problems affecting human heath, such as urban heat island effect, loss of biodiversity and enhanced $\mathrm{CO}_{2}$ emission (Ali et al., 2019; Bihamta et al., 2014; Dadashpoor \& Salarian, 2018; Min et al., 2019).

\section{Conclusions}

The major findings are as follows. (1) The landscape in Qingdao became less fragmented, less isolated and more aggregated under the urbanization from 1995 to 2010, evidenced by decreased PD, ED and SHDI and increased AI in urban core areas. This was due to the transformation from cultivated land, forest land and other land types to built-up land, which made land patches connected and more evenness. (2) The expanding block scale could reduce spatial differences of landscape metrics, decrease the total averaged patch density and aggregation and increase landscape diversity. (3) With the enlarging of block size, the explanatory ability of GWR models between landscape metrics and urbanization indicators generally increased, especially when it changed from $1 \mathrm{~km}$ to $5 \mathrm{~km}$ scale. When the block size was greater than $5 \mathrm{~km}$, GWR mostly performed well at the scale of odd-numbered than neighboring even-numbered. Furthermore, the metrics of $\mathrm{PD}, \mathrm{ED}, \mathrm{SHDI}$ and AI were all suitable for exploring spatial relationships between landscape pattern and land-use change.

Based on our study, some suggestions could be given when exploring spatial relationships between landscape pattern and urbanization using GWR. First, when the spatial resolution is $30 \mathrm{~m}$, block scale greater than $3 \mathrm{~km}$ could explore preferable models, especially those odd-numbered scale like $5 \mathrm{~km}, 7 \mathrm{~km}$ or $9 \mathrm{~km}$. Second, besides model performance improve, larger block scale could also reduce spatial difference of landscape metrics, so a tradeoff should be made between model performance and detailed landscape pattern exhibition. Finally, all of the four metrics and urbanization indicators used in this study were ideal for spatial relationship building.

\section{Acknowledgements}

We would like to thank the Data Center for Resources and Environmental Sciences, Chinese Academy of Sciences and Defense Meteorological Satellite Program for their assistance in providing data and information for the research. This research was financially supported by the Research Foundation for Advanced Talents, Qingdao Agricultural University (No. 663/1115007), Project of Forest
Resources Survey and Annual Forestland Updation (No. 20183702010498), and Construction Technology Research and Demonstration Project of Forest Landscape in Beautiful Towns (No. 201404301).

\section{Conflicts of interest}

The authors declare no conflict of interest.

\section{References}

Ali, R., Bakhsh, K., \& Yasin, M. A. (2019). Impact of urbanization on $\mathrm{CO}_{2}$ emissions in emerging economy: Evidence from Pakistan. Sustainable Cities and Society, 48, 101553. https://doi.org/10.1016/j.scs.2019.101553

Alibakhshi, Z., Ahmadi, M., \& Farajzadeh Asl, M. (2019). Modeling biophysical variables and land surface temperature using the GWR model: Case study-Tehran and its satellite cities. Journal of the Indian Society of Remote Sensing, 48, 59-70. https://doi.org/10.1007/s12524-019-01062-X

Barros, J., Tavares, A., Monteiro, M., \& Santos, P. (2018). PeriUrbanization and Rurbanization in Leiria City: The importance of a planning framework. Sustainability, 10(7), 2501. https://doi.org/10.3390/su10072501

Baugh, K., Elvidge, C. D., Ghosh, T., \& Ziskin, D. (2010). Development of a 2009 stable lights product using DMSP-OLS data. Proceedings of the Asia-Pacific Advanced Network Meeting. https://doi.org/10.7125/APAN.30.17

Bihamta, N., Soffianian, A., Fakheran, S., \& Gholamalifard, M. (2014). Using the SLEUTH urban growth model to simulate future urban expansion of the Isfahan Metropolitan Area, Iran. Journal of the Indian Society of Remote Sensing, 43(2), 407-414. https://doi.org/10.1007/s12524-014-0402-8

Dadashpoor, H., \& Salarian, F. (2018). Urban sprawl on natural lands: Analyzing and predicting the trend of land use changes and sprawl in Mazandaran city region, Iran. Environment, Development and Sustainability, 22, 593-614 (2020). https://doi.org/10.1007/s10668-018-0211-2

Dadashpoor, H., Azizi, P., \& Moghadasi, M. (2019). Land use change, urbanization, and change in landscape pattern in a metropolitan area. Science of the Total Environment, 655, 707-719. https://doi.org/10.1016/j.scitotenv.2018.11.267

Defense Meteorological Satellite Program (DMSP). (n.d.). National Centers for Environmental Information.

http://ngdc.noaa.gov/eog/dmsp.html

Deng, J. S., Wang, K., Hong, Y., \& Qi, J. G. (2009). Spatio-temporal dynamics and evolution of land use change and landscape pattern in response to rapid urbanization. Landscape and Urban Planning, 92(3-4), 187-198.

https://doi.org/10.1016/j.landurbplan.2009.05.001

Dormann, C. F., Elith, J., Bacher, S., Buchmann, C., Carl, G., Carré, G., \& Lautenbach, S. (2013). Collinearity: A review of methods to deal with it and a simulation study evaluating their performance. Ecography, 36(1), 27-46. https://doi.org/10.1111/j.1600-0587.2012.07348.x

Dormann, C. F., McPherson, J. M., Araújo, M. B., Bivand, R., Bolliger, J., Carl, G., Davies, R. G., Hirzel, A., Jetz, W., Daniel Kissling, W., Kühn, I., Ohlemüller, R., Peres-Neto, P. R., Reineking, B., Schröder, B., Schurr, F. M., \& Wilson, R. (2007). Methods to account for spatial autocorrelation in the analysis of species distributional data: A review. Ecography, 30(5), 609-628. https://doi.org/10.1111/j.2007.0906-7590.05171.x 
Du, S., Wang, Q., \& Guo, L. (2014). Spatially varying relationships between land-cover change and driving factors at multiple sampling scales. Journal of Environmental Management, 137, 101-110. https://doi.org/10.1016/j.jenvman.2014.01.037

Elvidge, D. C., Ziskin, D., Baugh, E. K., Tuttle, T. B., Ghosh, T., Pack, W. D., \& Zhizhin, M. (2009). A fifteen year record of global natural gas flaring derived from satellite data. Energies, 2(3), 595-622. https://doi.org/10.3390/en20300595

Feng, Y., \& Liu, Y. (2015). Fractal dimension as an indicator for quantifying the effects of changing spatial scales on landscape metrics. Ecological Indicators, 53, 18-27.

https://doi.org/10.1016/j.ecolind.2015.01.020

Feng, Y., Liu, Y., \& Tong, X. (2018). Spatiotemporal variation of landscape patterns and their spatial determinants in Shanghai, China. Ecological Indicators, 87, 22-32.

https://doi.org/10.1016/j.ecolind.2017.12.034

Fotheringham, A. S., Brunsdon, C., \& Charlton, M. E. (2002). Geographically weighted regression: The analysis of spatially varying relationships. Wiley.

Gao, J., \& Li, S. (2011). Detecting spatially non-stationary and scale-dependent relationships between urban landscape fragmentation and related factors using Geographically Weighted Regression. Applied Geography, 31(1), 292-302.

https://doi.org/10.1016/j.apgeog.2010.06.003

Herold, M., Goldstein, N. C., \& Clarke, K. C. (2003). The spatiotemporal form of urban growth: measurement, analysis and modeling. Remote Sensing of Environment, 86(3), 286-302. https://doi.org/10.1016/S0034-4257(03)00075-0

Herold, M., Scepan, J., \& Clarke, K. C. (2002). The Use of remote sensing and landscape metrics to describe structures and changes in urban land uses. Environment and Planning A: Economy and Space, 34(8), 1443-1458. https://doi.org/10.1068/a3496

Hou, L., Wu, F., \& Xie, X. (2020). The spatial characteristics and relationships between landscape pattern and ecosystem service value along an urban-rural gradient in Xian city, China. Ecological Indicators, 108, 105720.

https://doi.org/10.1016/j.ecolind.2019.105720

Jiao, M., Hu, M., \& Xia, B. (2019). Spatiotemporal dynamic simulation of land-use and landscape-pattern in the Pearl River Delta, China. Sustainable Cities and Society, 49, 101581. https://doi.org/10.1016/j.scs.2019.101581

Koch, J., Dorning, M. A., Van Berkel, D. B., Beck, S. M., Sanchez, G. M., Shashidharan, A., Smart, L. S., Zhang, Q., Smith, J. W., \& Meentemeyer, R. K. (2019). Modeling landowner interactions and development patterns at the urban fringe. Landscape and Urban Planning, 182, 101-113. https://doi.org/10.1016/j.landurbplan.2018.09.023

Li, C., \& Zhao, J. (2019). Investigating the spatiotemporally varying correlation between urban spatial patterns and ecosystem services: A case study of Nansihu Lake Basin, China. ISPRS International Journal of Geo-Information, 8(8), 346. https://doi.org/10.3390/ijgi8080346

Li, B., Chen, D., Wu, S., Zhou, S., Wang, T., \& Chen, H. (2016). Spatio-temporal assessment of urbanization impacts on ecosystem services: Case study of Nanjing City, China. Ecological Indicators, 71, 416-427. https://doi.org/10.1016/j.ecolind.2016.07.017

Li, F., Zheng, W., Wang, Y., Liang, J., Xie, S., Guo, S., Li, X., \& Yu, C. (2019). Urban green space fragmentation and urbanization: A spatiotemporal perspective. Forests, 10(4), 333. https://doi.org/10.3390/f10040333

Li, H., Peng, J., Yanxu, L., \& Yi'na, H. (2017). Urbanization impact on landscape patterns in Beijing City, China: A spatial heterogeneity perspective. Ecological Indicators, 82(Suppl C), 50-60. https://doi.org/10.1016/j.ecolind.2017.06.032

Li, S., Zhao, Z., Miaomiao, X., \& Wang, Y. (2010). Investigating spatial non-stationary and scale-dependent relationships between urban surface temperature and environmental factors using geographically weighted regression. Environmental Modelling \& Software, 25(12), 1789-1800.

https://doi.org/10.1016/j.envsoft.2010.06.011

Liu, Z., He, C., Zhang, Q., Huang, Q., \& Yang, Y. (2012). Extracting the dynamics of urban expansion in China using DMSPOLS nighttime light data from 1992 to 2008. Landscape and Urban Planning, 106(1), 62-72. https://doi.org/10.1016/j.landurbplan.2012.02.013

Luck, M., \& Wu, J. (2002). A gradient analysis of urban landscape pattern: A case study from the Phoenix metropolitan region, Arizona, USA. Landscape Ecology, 17(4), 327-339. https://doi.org/10.1023/A:1020512723753

Luo, X., \& Peng, Y. (2016). Scale effects of the relationships between urban heat islands and impact factors based on a geographically-weighted regression model. Remote Sensing, 8(9), 760. https://doi.org/10.3390/rs8090760

Mann, S. (2009). Institutional causes of urban and rural sprawl in Switzerland. Land Use Policy, 26(4), 919-924. https://doi.org/10.1016/j.landusepol.2008.11.004

Marull, J., Font, C., \& Boix, R. (2015). Modelling urban networks at mega-regional scale: Are increasingly complex urban systems sustainable? Land Use Policy, 43, 15-27. https://doi.org/10.1016/j.landusepol.2014.10.014

Min, M., Lin, C., Duan, X., Jin, Z., \& Zhang, L. (2019). Spatial distribution and driving force analysis of urban heat island effect based on raster data: A case study of the Nanjing metropolitan area, China. Sustainable Cities and Society, 50, 101637. https://doi.org/10.1016/j.scs.2019.101637

Peng, W., Wang, X., Li, X., \& He, C. (2018). Sustainability evaluation based on the emergy ecological footprint method: A case study of Qingdao, China, from 2004 to 2014. Ecological Indicators, 85, 1249-1261. https://doi.org/10.1016/j.ecolind.2017.12.020

Riitters, K. H., O’Neill, R. V., Hunsaker, C. T., Wickham, J. D., Yankee, D. H., Timmins, S. P., \& Jackson, B. L. (1995). A factor analysis of landscape pattern and structure metrics. Landscape Ecology, 10(1), 23-39. https://doi.org/10.1007/BF00158551

Shen, S., Yue, P., \& Fan, C. (2019). Quantitative assessment of land use dynamic variation using remote sensing data and landscape pattern in the Yangtze River Delta, China. Sustainable Computing: Informatics and Systems, 23, 111-119. https://doi.org/10.1016/j.suscom.2019.07.006

Shen, W., Jenerette, G. D., Wu, J., \& Gardner, R. H. (2004). Evaluating empirical scaling relations of pattern metrics with simulated landscapes. Ecography, 27(4), 459-469. https://doi.org/10.1111/j.0906-7590.2004.03799.x

Su, S., Jiang, Z., Zhang, Q., \& Zhang, Y. (2011). Transformation of agricultural landscapes under rapid urbanization: A threat to sustainability in Hang-Jia-Hu region, China. Applied Geography, 31(2), 439-449. https://doi.org/10.1016/j.apgeog.2010.10.008

Su, S., Ma, X., \& Xiao, R. (2014). Agricultural landscape pattern changes in response to urbanization at ecoregional scale. Ecological Indicators, 40, 10-18. https://doi.org/10.1016/j.ecolind.2013.12.013

Sun, X., Crittenden, J. C., Li, F., Lu, Z., \& Dou, X. (2018). Urban expansion simulation and the spatio-temporal changes of 
ecosystem services, a case study in Atlanta Metropolitan area, USA. Science of the Total Environment, 622-623, 974-987. https://doi.org/10.1016/j.scitotenv.2017.12.062

Tan, M., Li, X., Xie, H., \& Lu, C. (2005). Urban land expansion and arable land loss in China - a case study of Beijing-Tianjin-Hebei region. Land Use Policy, 22(3), 187-196. https://doi.org/10.1016/j.landusepol.2004.03.003

Tian, P., Cao, L., Li, J., Pu, R., Shi, X., Wang, L., \& Shao, S. (2019). Landscape grain effect in Yancheng Coastal Wetland and its response to landscape changes. International Journal of Environmental Research and Public Health, 16(12), 2225. https://doi.org/10.3390/ijerph16122225

Torres, A., Jaeger, J. A. G., \& Alonso, J. C. (2016). Multi-scale mismatches between urban sprawl and landscape fragmentation create windows of opportunity for conservation development. Landscape Ecology, 31(10), 2291-2305. https://doi.org/10.1007/s10980-016-0400-Z

Wang, K., Zhang, C., Chen, H., Yue, Y., Zhang, W., Zhang, M., Qi, X., \& Fu, Z. (2019). Karst landscapes of China: Patterns, ecosystem processes and services. Landscape Ecology, 34(12), 2743-2763. https://doi.org/10.1007/s10980-019-00912-w

Weng, Y.-C. (2007). Spatiotemporal changes of landscape pattern in response to urbanization. Landscape and Urban Planning, 81(4), 341-353.

https://doi.org/10.1016/j.landurbplan.2007.01.009

$\mathrm{Wu}, \mathrm{J}$. (2004). Effects of changing scale on landscape pattern analysis: Scaling relations. Landscape Ecology, 19(2), 125-138. https://doi.org/10.1023/B:LAND.0000021711.40074.ae

Wu, J., Shen, W., Sun, W., \& Tueller, P. T. (2002). Empirical patterns of the effects of changing scale on landscape metrics. Landscape Ecology, 17(8), 761-782. https://doi.org/10.1023/A:1022995922992
Xiao, H., Liu, Y., Li, L., Yu, Z., \& Zhang, X. (2018). Spatial variability of local rural landscape change under rapid urbanization in Eastern China. ISPRS International Journal of GeoInformation, 7(6), 231. https://doi.org/10.3390/ijgi7060231

$\mathrm{Xu}, \mathrm{C}$., Zhao, S., \& Liu, S. (2019). Spatial scaling of multiple landscape features in the conterminous United States. Landscape Ecology, 35, 223-247. https://doi.org/10.1007/s10980-019-00937-1

Xu, X., Liu, J., Zhang, S., Li, R., Yan, C., \& Wu, S. (2018). China’s land use remote sensing mapping system (CNLUCC) dataset. Resource and Environment Data cloud platform. http://www. resdc.cn/

Yang, J., Li, S., \& Lu, H. (2019). Quantitative influence of landuse changes and urban expansion intensity on landscape pattern in Qingdao, China: Implications for urban sustainability. Sustainability, 11(21), 6174. https://doi.org/10.3390/su11216174

Zhang, M., \& Rasiah, R. (2013). Qingdao. Cities, 31, 591-600. https://doi.org/10.1016/j.cities.2012.06.021

Zhang, Q., \& Seto, K. C. (2011). Mapping urbanization dynamics at regional and global scales using multi-temporal DMSP/ OLS nighttime light data. Remote Sensing of Environment, 115(9), 2320-2329. https://doi.org/10.1016/j.rse.2011.04.032

Zhang, Q., \& Su, S. (2016). Determinants of urban expansion and their relative importance: A comparative analysis of 30 major metropolitans in China. Habitat International, 58, 89107. https://doi.org/10.1016/j.habitatint.2016.10.003

Zhou, W., \& Cao, F. (2020). Effects of changing spatial extent on the relationship between urban forest patterns and land surface temperature. Ecological Indicators, 109, 105778. https://doi.org/10.1016/j.ecolind.2019.105778 